\title{
Soil Carbon Dynamics in Oil Palm - Cocoa Cropping System in Comparison to that of Oil Palm Sole Crop
}

\author{
Manoja Katakam and K. Suresh* \\ ICAR - Indian Institute of Oil Palm Research, Pedavegi - 534 450, Andhra Pradesh, India \\ *Corresponding author
}

\section{A B S T R A C T}

\begin{tabular}{|c|}
\hline $\begin{array}{l}\text { K e y w o r d s } \\
\text { carbon dynamics, } \\
\text { cocoa, cropping } \\
\text { system, oil palm, } \\
\text { organic carbon }\end{array}$ \\
\hline Article Info \\
\hline $\begin{array}{l}\text { Accepted: } \\
20 \text { February } 2020 \\
\text { Available Online: } \\
10 \text { March } 2020\end{array}$ \\
\hline
\end{tabular}

An investigation was conducted in oil palm sole crop and oil palm + cocoa cropping systems to study microbial diversity along with enzyme activities and understand soil carbon dynamics in oil palm based cropping system. Ten mature oil palm plantations (five each in oil palm sole crop and oil palm + cocoa) planted with Tenera hybrids with a spacing of $9 \times 9 \times 9 \mathrm{~m}$ in a triangular system were selected for the study. Cocoa var. Forastero is cultivated as an intercrop in oil palm plantations at $4.5 \times 4.5 \mathrm{~m}$ spacing. Recommended package of practices for oil palm and cocoa cultivation were followed. Soil samples were collected in the two cropping systems (oil palm sole crop and oil palm + cocoa) at three consecutive depths from surface $(0-15,15-30 \& 30-60 \mathrm{~cm})$ and five distances from palm base $(1,2,3,4 \& 5$ meters) during the three seasons (summer, winter \& rainy). The collected samples of soil were evaluated for soil carbon (C) dynamics viz., organic carbon $(\mathrm{OC})$, potassium permanganate extractable $\mathrm{C}$, readily mineralizable $\mathrm{C}$, acid hydrolysable $\mathrm{C}$, soluble $\mathrm{C}$ and microbial biomass $\mathrm{C}$. Studies on soil carbon dynamics indicated that all the organic carbon fractions of two cropping systems varied significantly and highest values were recorded under oil palm + cocoa system. Higher carbon fractions were observed in summer, while lower fractions were seen during winter and rainy periods. Significant differences in soil carbon fractions were observed among three soil layers viz., 0 to 15,15 to $30 \& 30$ to $60 \mathrm{~cm}$. All the carbon fractions decreased from surface to bottom soil layers. A linear increment in soil carbon fractions was observed with increase in distance from palm base.

\section{Introduction}

The role of plant and soils as a carbon sink is of vital significance in mitigating global warming effects and rising greenhouse gases in the atmosphere. Plantation crops offer tremendous scope for enhancing the carbon pool on the land surface. Among the plantation crops, oil palm is an important crop in India grown in 0.34 million ha. The crop is being grown in India decrease the supply and demand of vegetable oils in the country.

Oil palm is being cultivated as a small holders' crop in India both under rainfed and irrigated conditions (Suresh and Kiran kumar, 2011). In order to supplement the income, cocoa is being grown as a successful intercrop in adult oil palm plantations (6-8 years) by majority of oil palm farmers. 
Oil palm + cocoa cropping system is one of the finest models in terms of efficient utilization of horizontal and vertical space, profitability and sustainability under Indian conditions. Oil palm + cocoa cropping systems are effective in improving soil quality by continuous deposition of plant litter from cocoa and turnover of litter offers constant enrichment of organic matter to the soil through microbial activities (Bhagya and Suresh, 2018). However, studies on interactions among different biota in soil, organic $\mathrm{C}$ fractions along with enzymes in soils of oil palm + cocoa cropping system are very limited under irrigated conditions.

Knowledge of the dynamics between soil biota and microbial biomass carbon could play a vital role in understanding the oil palm + cocoa cropping system. Soil biota symbolizes a huge proportion of distribution in the earth's biodiversity. Deciphering the soil microbial activities has helped in getting valuable information of the ecosystems. Microorganisms in soils might be vital in affecting soil biological and chemical features (Shrinivas et al., 2019).

The association concerning carbon fractions and microorganisms in soils are multi-faceted. This interaction is critical in gaining knowledge on carbon sequestration and stability in soils. Variations in spatial distribution of microorganisms in soils largely influences turnover of carbon by interacting with crops and soil chemical properties. Land use changes could affect the capacity of soil to sequester carbon as it affects a radical shift in yield and carbon turn over (Gileno V. Lacerda-Júniorb et al., 2019). Studies pertaining to soil carbon dynamics in oil palm + cocoa based cropping system are very scarce, the present research was undertaken to understand soil carbon dynamics in oil palm based cropping system.

\section{Materials and Methods}

The study was taken up in ten mature oil palm plantations (5 with oil palm sole crop and 5 with oil palm + cocoa) belonging to farmers of Pedavegi mandal, West Godavari Dt., Andhra Pradesh, India. Pedavegi is situated at $16.80^{\circ} \mathrm{N}$ latitude, $81.11^{\circ} \mathrm{E}$ longitude and 85 $\mathrm{m}$ above mean sea level. The average temperature ranged from minimum of $24-27^{0}$ $\mathrm{C}$ to maximum of $34-36^{\circ} \mathrm{C}$ with an average rainfall of $648 \mathrm{~mm}$. All the oil palm plantations were prior planted with tenera hybrids with a spacing of $9 \times 9 \times 9 \mathrm{~m}$ in a triangular system. Cocoa var. Forastero is cultivated as an intercrop in oil palm plantations at a spacing of $4.5 \times 4.5 \mathrm{~m}$. Plantations having uniform age, yield and management practices have been selected for the study. Recommended management practices for oil palm and cocoa cultivation were followed. The soils in the study area were alluvial soils. Soil samples were collected during 2010-12 in the two cropping systems (oil palm sole crop and oil palm + cocoa) at three consecutive depths from surface $(0$ to $15 \mathrm{~cm}, 15$ to $30 \mathrm{~cm}$ and 30 to 60 $\mathrm{cm})$ and at five distances from palm base (1 metre, 2 metre, 3 metre, 4 metre, and 5 metre) during the three seasons (summer, winter and rainy).

Soil sampling was done by triangular sampling method as per Tailliez (1971) by using an auger (Chan, 1976) in all the 10 plantations under study. The triangle was sub divided into 16 sub triangles with sides 2.20 meters and from each centre of sub triangle, one core of sample were taken with the help of augur (10 cm diameter). Circular soil cores were collected in the two cropping systems (oil palm sole crop and oil palm + cocoa) at three consecutive depths from surface ( 0 to15 $\mathrm{cm}, 15$ to $30 \mathrm{~cm}$ and 30 to $60 \mathrm{~cm}$ ) and at five distances from palm base (1 to $5 \mathrm{~m}$ ) during the three seasons (summer, winter and rainy). 
Two sets of samples were collected by quartering technique from three triangles in each plantation. Immediately after sampling, excess water was drained off and visible root fragments were separated and stones removed manually. One set of fresh samples was used for estimating enzymes and microbial diversity studies and the other was air dried, sieved with $2 \mathrm{~mm}$ sieve and stored in polythene bags for estimation of Organic carbon (OC), $\mathrm{KMnO}_{4}$ extractable carbon, Acid hydrolysable $\mathrm{C}$, Microbial biomass $\mathrm{C}$, Readily mineralizable $\mathrm{C}$, and Water soluble C. The soil organic carbon content $(\%)$ was estimated as per Walkley and Black's, 1934. Readily mineralizable C (RMC) and Microbial biomass C (MBC) was estimated as per Vance et al., 1987. WSC content was estimated as per W. B. McGill et. al., 1986. The potassium permanganate extractable carbon was estimated as per Blair et al., 1995. Acid hydrolysable carbon was estimated as per Cheshire and Mundie, 1966. The statistical design used was four factorial completely randomised block design and data was analysed as per Gomez and Gomez, 1984.

\section{Results and Discussion}

The microbial carbon (MBC),WSC, organic carbon (SOC), AHC, readily mineralizable carbon (RMC) and POXC contents of two cropping systems (oil palm sole crop and oil palm + cocoa) varied significantly and highest values were recorded under oil palm + cocoa system (Tables 1-6). Different soil properties, climate, type of forest, litter quality and litter production by vegetation influence the soil carbon content (Don et al., 2007). The average carbon contents in surface soil layers were less in cultivated environments compared to that of mixed and natural forest land use situations (Amanuel et al., 2018). In alley cropping systems of tropical regions, significant changes of microbial biomass were found (Stefan Seiter et al., 1999). Mersch et al., 1993 also reported larger amounts of microbial mass in alley systems compared to that of single cropping situations. Kachaka et al., 1993 also recorded that higher biomass could be seen in alley situations compared to that of controlled environments. Forest soils possessed greater amounts of organic matter compared to that of paddy and dry environments (Jendoubi et al., 2019).

During the seasons, higher contents of POXC, AHC, SOC, RMC, MBC and WSC were recorded during summer in comparison to that other seasons studied (Tables 1-6). Interaction among different seasons and cropping systems revealed that the organic carbon content differed significantly during different seasons in two cropping systems. Similar trend was observed in oil palm sole crop also.

This is in tune with that of Singh and Yadava, 2006, who reported higher amounts during may and lower amounts during September. During summer season high organic nutrients and moderate temperature promote the microbial activity. This is in tune with that of Srivastava \& Singh, 1991, who have reported higher amounts in summer in grasslands of tropical conditions of India. The samples collected during May usually record high bacterial biomass values which might be due to peak microbial growth that occurs in spring. Bacterial biomass will be higher during May to July (Stefan Seiter 1999).

Ryan et al., 2009 noticed higher organic matter and permanganate-oxidizable carbon in fall and winter. It has been reported that biomass carbon has been greater in summer (wet) compared to that of winter (dry) season in rubber plantations, (Pan et al., 2000). Significant variations in $\mathrm{C}$ fractions estimated were seen in soil layers. The amounts of $\mathrm{C}$ fractions were greatest in surface layers and lower contents were recorded in bottom most soil layers (Tables 1-6). 
Table.1 Variations in organic carbon (units) in soils of Oil Palm sole crop and Oil Palm + Cocoa plantations at different depths and distances from palm base during various seasons

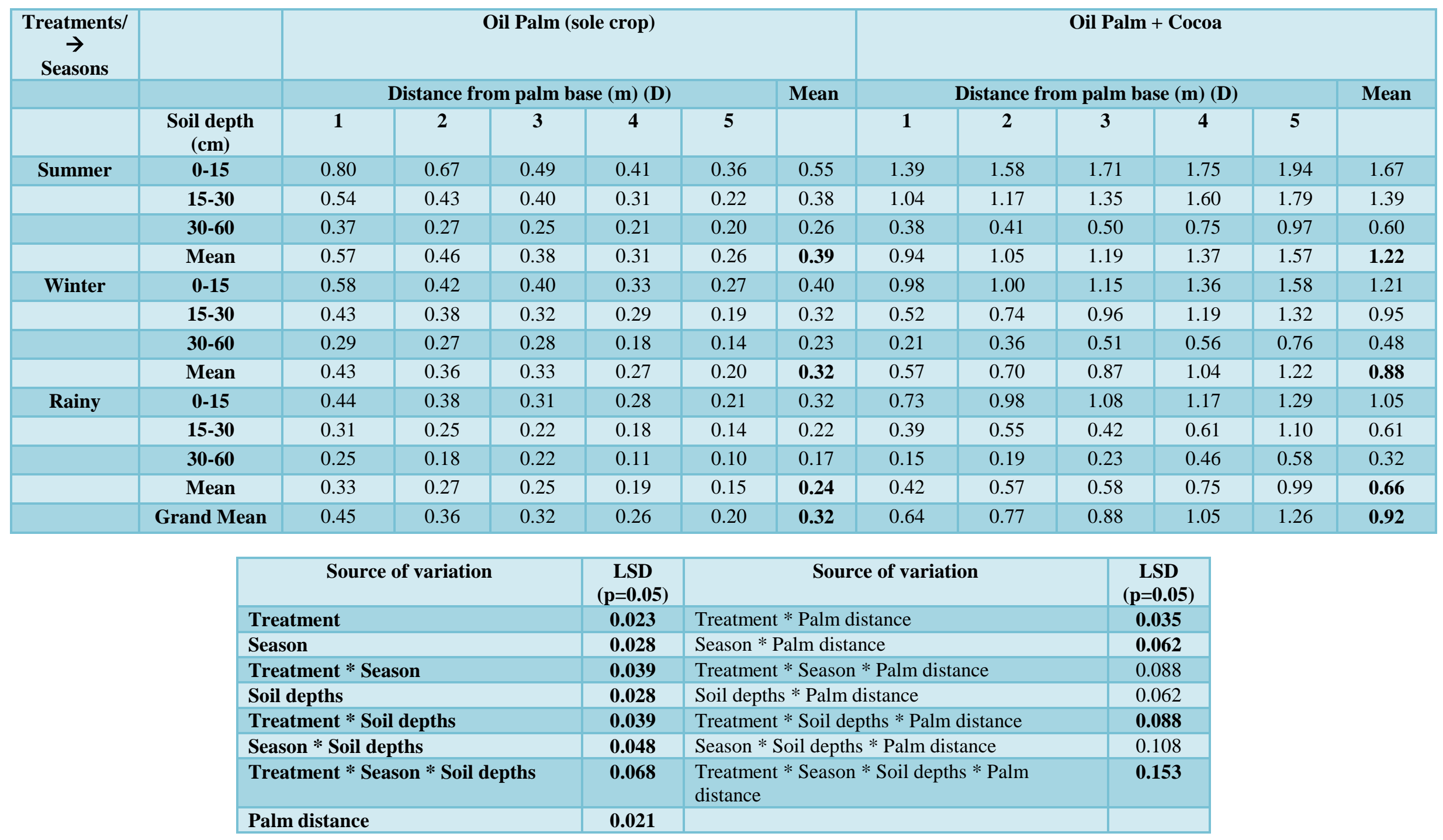


Table.2 Variations in Microbial Biomass Carbon (MBC-2) in soils of Oil Palm sole crop and Oil Palm + Cocoa plantations at different depths and distances from palm base during various seasons

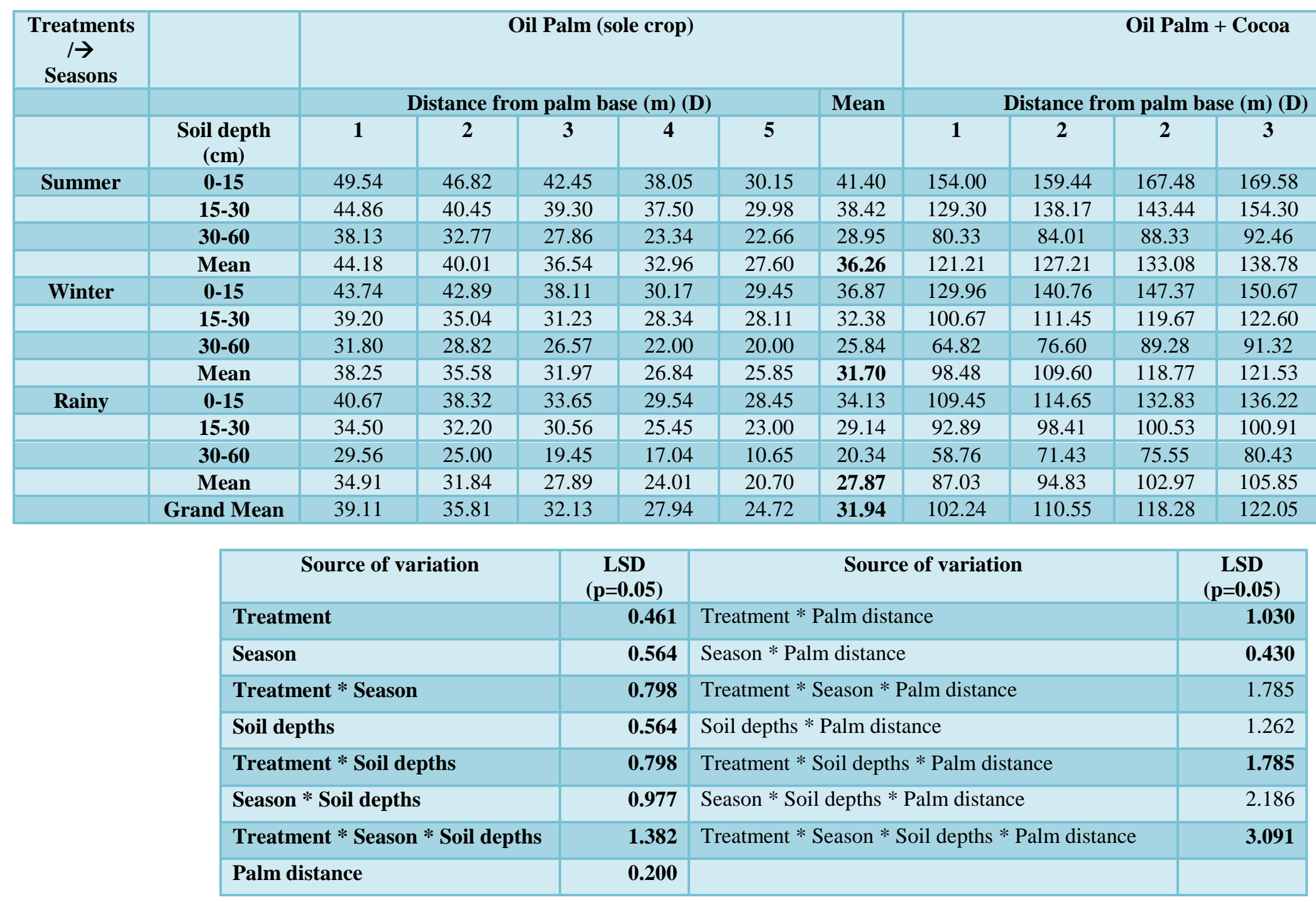


Table.3 Variations in Water Soluble Carbon (WSC) in soils of Oil Palm sole crop and Oil Palm + Cocoa plantations at different depths and distances from palm base during various seasons

\begin{tabular}{|c|c|c|c|c|c|c|c|c|c|c|c|c|c|}
\hline \multirow{3}{*}{$\begin{array}{c}\text { Treatment } \\
\text { s/ } \rightarrow \\
\text { Seasons }\end{array}$} & \multirow[b]{3}{*}{$\begin{array}{l}\text { Soil depth } \\
\quad(\mathrm{cm})\end{array}$} & \multicolumn{6}{|c|}{ Oil Palm (sole crop) } & \multicolumn{6}{|c|}{ Oil Palm + Cocoa } \\
\hline & & \multicolumn{5}{|c|}{ Distance from palm base (m) (D) } & \multirow[t]{2}{*}{ Mean } & \multicolumn{5}{|c|}{ Distance from palm base (m) (D) } & \multirow[t]{2}{*}{ Mean } \\
\hline & & 1 & 2 & 3 & 4 & 5 & & 1 & 2 & 3 & 4 & 5 & \\
\hline \multirow[t]{4}{*}{ Summer } & 0-15 & 32.66 & 29.34 & 26.31 & 26.49 & 21.98 & 27.36 & 31.00 & 37.00 & 39.65 & 42.68 & 60.34 & 42.13 \\
\hline & $15-30$ & 28.12 & 24.55 & 22.50 & 25.22 & 19.18 & 23.92 & 31.68 & 35.62 & 38.77 & 37.45 & 55.00 & 39.70 \\
\hline & $30-60$ & 20.75 & 19.57 & 18.43 & 15.28 & 11.34 & 17.07 & 29.56 & 30.55 & 31.05 & 30.16 & 38.45 & 31.95 \\
\hline & Mean & 27.18 & 24.49 & 22.41 & 22.33 & 17.50 & 22.78 & 30.75 & 34.39 & 36.49 & 36.76 & 51.26 & 37.93 \\
\hline \multirow[t]{4}{*}{ Winter } & $0-15$ & 26.00 & 24.00 & 23.62 & 21.27 & 18.32 & 22.64 & 27.31 & 30.66 & 34.00 & 44.34 & 46.34 & 36.53 \\
\hline & $15-30$ & 17.53 & 17.48 & 15.73 & 13.47 & 12.55 & 15.35 & 20.21 & 24.30 & 26.45 & 37.82 & 37.87 & 29.33 \\
\hline & $30-60$ & 15.84 & 13.34 & 11.61 & 12.33 & 10.33 & 12.69 & 19.11 & 20.14 & 22.30 & 33.68 & 36.18 & 26.28 \\
\hline & Mean & 19.79 & 18.27 & 16.99 & 15.69 & 13.73 & 16.90 & 22.21 & 25.03 & 27.58 & 38.61 & 40.13 & 30.71 \\
\hline \multirow[t]{5}{*}{ Rainy } & $0-15$ & 18.99 & 16.75 & 16.05 & 17.47 & 10.68 & 15.99 & 31.02 & 37.81 & 36.39 & 37.09 & 39.33 & 36.33 \\
\hline & $15-30$ & 16.80 & 15.67 & 14.46 & 12.30 & 10.00 & 13.85 & 15.01 & 15.08 & 19.08 & 25.01 & 28.44 & 20.52 \\
\hline & $30-60$ & 13.54 & 10.66 & 12.67 & 10.66 & 9.56 & 11.42 & 12.04 & 12.89 & 15.77 & 20.15 & 27.19 & 17.61 \\
\hline & Mean & 16.44 & 14.36 & 14.39 & 13.47 & 10.08 & 13.75 & 19.36 & 21.93 & 23.75 & 27.42 & 31.65 & 24.82 \\
\hline & Grand Mean & 21.14 & 19.04 & 17.93 & 17.17 & 13.77 & 17.81 & 24.10 & 27.12 & 29.27 & 34.26 & 41.02 & 31.15 \\
\hline
\end{tabular}

\begin{tabular}{|c|c|c|c|}
\hline Source of variation & $\begin{array}{c}\text { LSD } \\
(p=0.05)\end{array}$ & Source of variation & $\begin{array}{c}\text { LSD } \\
(p=0.05)\end{array}$ \\
\hline Treatment & 0.443 & Treatment $*$ Palm distance & 0.679 \\
\hline Season & 0.543 & Season * Palm distance & 0.125 \\
\hline Treatment $*$ Season & 0.768 & Treatment $*$ Season $*$ Palm distance & 1.716 \\
\hline Soil depths & 0.543 & Soil depths $*$ Palm distance & 1.214 \\
\hline Treatment $*$ Soil depths & 0.768 & Treatment $*$ Soil depths $*$ Palm distance & 1.716 \\
\hline Season * Soil depths & 0.940 & Season $*$ Soil depths $*$ Palm distance & 2.102 \\
\hline Treatment $*$ Season $*$ Soil depths & 1.001 & Treatment $*$ Season $*$ Soil depths $*$ Palm distance & 2.973 \\
\hline Palm distance & 0.444 & & \\
\hline
\end{tabular}


Table.4 Variations in Permanganate soluble carbon (POSC) in soils of Oil Palm sole crop and Oil Palm + Cocoa plantations at different depths and distances from palm base during various seasons

\begin{tabular}{|c|c|c|c|c|c|c|c|c|c|c|c|c|c|}
\hline \multirow{3}{*}{$\begin{array}{c}\text { Treatments/ } \\
\rightarrow \\
\text { Seasons }\end{array}$} & \multirow[b]{3}{*}{ Soil depth (cm) } & \multicolumn{6}{|c|}{ Oil Palm (sole crop) } & \multicolumn{6}{|c|}{ Oil Palm + Cocoa } \\
\hline & & \multicolumn{5}{|c|}{ Distance from palm base (m) (D) } & \multirow[t]{2}{*}{ Mean } & \multicolumn{5}{|c|}{ Distance from palm base (m) (D) } & \multirow[t]{2}{*}{ Mean } \\
\hline & & 1 & 2 & 3 & 4 & 5 & & 1 & 2 & 3 & 4 & 5 & \\
\hline \multirow[t]{4}{*}{ Summer } & 0-15 & 388.44 & 378.34 & 371.09 & 364.00 & 340.99 & 368.57 & 429.76 & 453.78 & 461.04 & 467.95 & 478.39 & 458.18 \\
\hline & $15-30$ & 369.67 & 363.65 & 360.00 & 355.87 & 336.76 & 357.19 & 427.00 & 446.00 & 450.76 & 454.98 & 459.55 & 447.66 \\
\hline & $30-60$ & 208.00 & 200.00 & 195.44 & 191.65 & 185.89 & 196.20 & 324.09 & 331.22 & 335.43 & 340.000 & 348 & 335.75 \\
\hline & Mean & 322.04 & 314.00 & 308.84 & 303.84 & 287.88 & 307.32 & 393.62 & 410.33 & 415.74 & 420.98 & 428.65 & 413.86 \\
\hline \multirow[t]{4}{*}{ Winter } & $0-15$ & 376.45 & 367.31 & 360.09 & 353.00 & 329.61 & 357.29 & 380.00 & 404.31 & 411.09 & 418.03 & 428.44 & 408.37 \\
\hline & $15-30$ & 357.87 & 352.76 & 348.43 & 345.01 & 324.98 & 345.81 & 377.23 & 396.34 & 400.65 & 404.11 & 409.45 & 397.56 \\
\hline & $30-60$ & 197.54 & 189.22 & 184.56 & 180.88 & 173.02 & 185.04 & 225.44 & 231.98 & 235.28 & 240.24 & 248.00 & 236.19 \\
\hline & Mean & 310.62 & 303.10 & 297.69 & 292.96 & 275.87 & 296.05 & 327.56 & 344.21 & 349.01 & 354.13 & 361.96 & $\mathbf{3 4 7 . 3 7}$ \\
\hline \multirow[t]{5}{*}{ Rainy } & $0-15$ & 263.44 & 253.00 & 246.09 & 239.00 & 215.32 & 243.37 & 260.03 & 284.78 & 291.09 & 298.26 & 308.31 & 288.49 \\
\hline & $15-30$ & 244.87 & 239.05 & 235.78 & 230.64 & 212.00 & 232.47 & 257.31 & 276.15 & 280.00 & 284.25 & 289.33 & 277.41 \\
\hline & $30-60$ & 143.08 & 135.91 & 130.00 & 126.04 & 119.08 & 130.82 & 204.00 & 211.56 & 215.20 & 220.98 & 228.00 & 215.95 \\
\hline & Mean & 217.13 & 209.32 & 203.96 & 198.56 & 182.13 & 202.22 & 240.45 & 257.50 & 262.10 & 267.83 & 275.21 & 260.62 \\
\hline & Grand Mean & 283.26 & 275.47 & 270.16 & 265.12 & 248.63 & 268.53 & 320.54 & 337.35 & 342.28 & 347.64 & 355.27 & 340.62 \\
\hline
\end{tabular}

\begin{tabular}{|l|c|l|c|}
\hline \multicolumn{1}{|c|}{ Source of variation } & $\begin{array}{c}\text { LSD } \\
(\mathbf{p = 0 . 0 5 )}\end{array}$ & \multicolumn{1}{|c|}{$\begin{array}{c}\text { Source of variation } \\
(\mathbf{p = 0 . 0 5})\end{array}$} \\
\hline Treatment & $\mathbf{1 . 7 6 1}$ & Treatment * Palm distance & $\mathbf{1 . 9 5 6}$ \\
\hline Season & $\mathbf{2 . 1 4 1}$ & Season * Palm distance & $\mathbf{0 . 1 0 0}$ \\
\hline Treatment * Season & $\mathbf{2 . 9 9 8}$ & Treatment * Season * Palm distance & 2.147 \\
\hline Soil depths & $\mathbf{2 . 1 4 1}$ & Soil depths * Palm distance & 1.762 \\
\hline Treatment * Soil depths & $\mathbf{2 . 9 9 8}$ & Treatment * Soil depths * Palm distance & $\mathbf{1 . 3 7 8}$ \\
\hline Season * Soil depths & $\mathbf{3 . 6 5 5}$ & Season * Soil depths * Palm distance & 2.076 \\
\hline Treatment * Season * Soil depths & $\mathbf{2 . 0 1 8}$ & Treatment * Season * Soil depths * Palm distance \\
\hline Palm distance & $\mathbf{0 . 0 2 4}$ & & $\mathbf{1 . 6 2 1}$ \\
\hline
\end{tabular}


Table.5 Variations in Acid Hydrosoluble carbon (AHC) in soils of Oil Palm sole crop and Oil Palm + Cocoa plantations at different depths and distances from palm base during various seasons

\begin{tabular}{|c|c|c|c|c|c|c|c|c|c|c|c|c|c|}
\hline \multirow{3}{*}{$\begin{array}{c}\text { Treatmen } \\
\text { ts } / \rightarrow \\
\text { Seasons }\end{array}$} & & \multicolumn{6}{|c|}{ Oil Palm (sole crop) } & \multicolumn{6}{|c|}{ Oil Palm + Cocoa } \\
\hline & \multirow[b]{2}{*}{$\begin{array}{l}\text { Soil depth } \\
\text { (cm) }\end{array}$} & \multicolumn{5}{|c|}{ Distance from palm base (m) (D) } & \multirow[t]{2}{*}{ Mean } & \multicolumn{5}{|c|}{ Distance from palm base (m) (D) } & \multirow[t]{2}{*}{ Mean } \\
\hline & & 1 & 2 & 3 & 4 & 5 & & 1 & 2 & 3 & 4 & 5 & \\
\hline \multirow[t]{4}{*}{ Summer } & 0-15 & 188.44 & 178.34 & 171.09 & 164.00 & 140.99 & 168.57 & 229.76 & 253.78 & 261.04 & 267.95 & 278.39 & 258.18 \\
\hline & 15-30 & 169.67 & 163.65 & 160.00 & 155.87 & 136.76 & 157.19 & 227.00 & 246.00 & 250.76 & 254.98 & 259 & 247.55 \\
\hline & $30-60$ & 108.00 & 100.00 & 95.44 & 91.65 & 85.89 & 96.20 & 174.09 & 181.22 & 185.43 & 190.000 & 198 & 185.75 \\
\hline & Mean & 155.37 & 147.33 & 142.18 & 137.17 & 121.21 & 140.65 & 210.28 & 227.00 & 232.41 & 237.64 & 245.13 & 230.49 \\
\hline \multirow[t]{4}{*}{ Winter } & $0-15$ & 176.45 & 167.31 & 160.09 & 153.00 & 129.61 & 157.29 & 180.00 & 204.31 & 211.09 & 218.03 & 228.44 & 208.37 \\
\hline & $15-30$ & 157.87 & 152.76 & 148.43 & 145.01 & 124.98 & 145.81 & 177.23 & 196.34 & 200.65 & 204.11 & 209.45 & 197.56 \\
\hline & $30-60$ & 97.54 & 89.22 & 84.00 & 80.88 & 73.02 & 84.93 & 125.44 & 131.98 & 135.28 & 140.24 & 148.00 & 136.19 \\
\hline & Mean & 143.95 & 136.43 & 130.84 & 126.30 & 109.20 & 129.34 & 160.89 & 177.54 & 182.34 & 187.46 & 195.30 & 180.71 \\
\hline \multirow[t]{5}{*}{ Rainy } & $0-15$ & 163.44 & 153.00 & 146.09 & 139.00 & 115.32 & 143.37 & 160.03 & 184.78 & 191.09 & 198.26 & 208.31 & 188.49 \\
\hline & $15-30$ & 144.87 & 139.05 & 135.78 & 130.64 & 112.00 & 132.47 & 157.31 & 176.15 & 180.00 & 184.25 & 189.33 & 177.41 \\
\hline & $30-60$ & 93.08 & 85.91 & 80.00 & 76.00 & 69.08 & 80.81 & 104.00 & 111.56 & 115.20 & 120.00 & 128.00 & 115.75 \\
\hline & Mean & 133.80 & 125.99 & 120.62 & 115.21 & 98.80 & 118.88 & 140.45 & 157.50 & 162.10 & 167.50 & 175.21 & 160.55 \\
\hline & $\begin{array}{l}\text { Grand } \\
\text { Mean }\end{array}$ & 144.37 & 136.58 & 131.21 & 126.23 & 109.74 & 129.63 & 170.54 & 187.35 & 192.28 & 197.54 & 205.21 & 190.58 \\
\hline
\end{tabular}

\begin{tabular}{|c|c|c|c|}
\hline Source of variation & $\begin{array}{c}\text { LSD } \\
(p=0.05)\end{array}$ & Source of variation & $\begin{array}{c}\text { LSD } \\
(p=0.05)\end{array}$ \\
\hline Treatment & 1.688 & Treatment $*$ Palm distance & 3.776 \\
\hline Season & 2.068 & Season * Palm distance & 1.256 \\
\hline Treatment $*$ Season & 2.925 & Treatment $*$ Season $*$ Palm distance & 2.074 \\
\hline Soil depths & 2.068 & Soil depths * Palm distance & 1.689 \\
\hline Treatment $*$ Soil depths & 0.689 & Treatment $*$ Soil depths $*$ Palm distance & 1.305 \\
\hline Season $*$ Soil depths & 3.582 & Season * Soil depths * Palm distance & 2.003 \\
\hline Treatment $*$ Season $*$ Soil depths & 1.945 & Treatment $*$ Season $*$ Soil depths $*$ Palm distance & 1.548 \\
\hline Palm distance & 2.670 & & \\
\hline
\end{tabular}


Table.6 Variations in Readily mineralizable carbon (RMC) in soils of Oil Palm sole crop and Oil Palm + Cocoa plantations at different depths and distances from palm base during various seasons

\begin{tabular}{|c|c|c|c|c|c|c|c|c|c|c|c|c|c|}
\hline \multirow{3}{*}{$\begin{array}{c}\text { Treatments } \\
\qquad \rightarrow \\
\text { Seasons }\end{array}$} & & \multicolumn{6}{|c|}{ Oil Palm (sole crop) } & \multicolumn{6}{|c|}{ Oil Palm + Cocoa } \\
\hline & \multirow[b]{2}{*}{$\begin{array}{l}\text { Soil depth } \\
\text { (cm) }\end{array}$} & \multicolumn{5}{|c|}{ Distance from palm base (m) (D) } & \multirow[t]{2}{*}{ Mean } & \multicolumn{5}{|c|}{ Distance from palm base (m) (D) } & \multirow[t]{2}{*}{ Mean } \\
\hline & & 1 & 2 & 3 & 4 & 5 & & 1 & 2 & 3 & 4 & 5 & \\
\hline \multirow[t]{4}{*}{ Summer } & 0-15 & 45.73 & 43.04 & 38.68 & 34.27 & 26.38 & 37.62 & 65.38 & 73.28 & 77.68 & 83.12 & 84.77 & 76.85 \\
\hline & $15-30$ & 41.14 & 36.62 & 35.54 & 34.25 & 26.19 & 34.75 & 64.20 & 73.23 & 74.53 & 75.68 & 80.09 & 73.55 \\
\hline & $30-60$ & 34.33 & 28.93 & 24.06 & 19.57 & 18.86 & 25.15 & 57.89 & 58.57 & 63.09 & 68.00 & 73.36 & 64.18 \\
\hline & Mean & 40.40 & 36.20 & 32.76 & 29.36 & 23.81 & 32.51 & 62.49 & 68.36 & 71.77 & 75.60 & 79.41 & 71.53 \\
\hline \multirow[t]{4}{*}{ Winter } & $0-15$ & 39.56 & 39.06 & 34.31 & 26.37 & 25.65 & 32.99 & 60.68 & 61.40 & 69.34 & 74.12 & 74.57 & 68.02 \\
\hline & $15-30$ & 35.40 & 31.26 & 27.43 & 24.56 & 24.31 & 28.59 & 55.45 & 58.68 & 62.46 & 66.27 & 70.43 & 62.66 \\
\hline & $30-60$ & 28.00 & 25.02 & 22.77 & 18.20 & 16.22 & 22.04 & 52.00 & 53.23 & 57.80 & 60.05 & 63.03 & 57.22 \\
\hline & Mean & 34.32 & 31.78 & 28.17 & 23.04 & 22.06 & 27.87 & 56.04 & 57.77 & 63.20 & 66.81 & 69.34 & 62.63 \\
\hline \multirow[t]{5}{*}{ Rainy } & $0-15$ & 36.89 & 34.52 & 29.85 & 25.74 & 24.65 & 30.33 & 60.68 & 61.77 & 65.88 & 70.55 & 72.90 & 66.36 \\
\hline & $15-30$ & 30.70 & 28.41 & 26.77 & 21.65 & 19.20 & 25.35 & 45.33 & 57.68 & 62.79 & 64.43 & 66.73 & 59.39 \\
\hline & $30-60$ & 25.76 & 21.27 & 15.65 & 13.24 & 6.98 & 16.58 & 33.55 & 49.27 & 51.68 & 57.23 & 61.79 & 50.70 \\
\hline & Mean & 31.12 & 28.07 & 24.09 & 20.21 & 16.94 & 24.08 & 46.52 & 56.24 & 60.12 & 64.07 & 67.14 & 58.82 \\
\hline & Grand Mean & 35.28 & 32.01 & 28.34 & 24.21 & 20.94 & 28.16 & 55.02 & 60.79 & 65.03 & 68.83 & 71.96 & 64.33 \\
\hline
\end{tabular}

\begin{tabular}{|l|r|l|r|}
\hline \multicolumn{1}{|c|}{ Source of variation } & $\begin{array}{c}\text { LSD } \\
(\mathbf{p = 0 . 0 5 )}\end{array}$ & \multicolumn{1}{|c|}{ Source of variation } \\
$(\mathbf{p = 0 . 0 5})$ \\
\hline Treatment & $\mathbf{0 . 6 0 3}$ & Treatment * Palm distance & $\mathbf{1 . 3 4 7}$ \\
\hline Season & $\mathbf{0 . 7 3 8}$ & Season * Palm distance & $\mathbf{1 . 6 5 0}$ \\
\hline Treatment * Season & $\mathbf{1 . 0 4 4}$ & Treatment * Season * Palm distance & 1.876 \\
\hline Soil depths & $\mathbf{0 . 7 3 8}$ & Soil depths * Palm distance & 1.650 \\
\hline Treatment * Soil depths & $\mathbf{1 . 0 4 4}$ & Treatment * Soil depths * Palm distance & 1.56 \\
\hline Season * Soil depths & $\mathbf{1 . 2 7 8}$ & Season * Soil depths * Palm distance & 1.540 \\
\hline Treatment * Season * Soil depths & $\mathbf{1 . 8 0 8}$ & Treatment * Season * Soil depths * Palm distance \\
\hline Palm distance & $\mathbf{0 . 0 3 5}$ & & $\mathbf{0 . 2 4 5}$ \\
\hline
\end{tabular}


Similar patterns were detected in oil palm sole crop and oil palm + cocoa system. At different soil depths, all $\mathrm{C}$ fractions were greater in oil palm + cocoa system than oil palm sole crop. Similarility of results could be seen with that of vanStraaten $\mathrm{O}$ et al., 2015. In soil layers studied, OM has been significantly higher in cover crops compared to no cover crops (Lalith M. Rankoth et al., 2019). The amounts of $C$ fractions were higher in surface soils compared to that bottom soil layers, which is in tune with Zhang et al., 2019. The top $30 \mathrm{~cm}$ of soil surface hold approximately 52 per cent of carbon pool and subjected to changes with change in land use pattern and deforestation.

A linear increment with respect to $\mathrm{C}$ fractions was observed with increase in distance from palms (Tables 1-6). Maximum contents of $C$ fractions content was noticed at $5 \mathrm{~m}$ distance from palms and decreased gradually with decrease in distance from palms. Highest $\mathrm{SOC}$, WSC was at $5 \mathrm{~m}$ distance from palm base in oil palm + cocoa system, while lowest was in oil palm sole crop at $1 \mathrm{~m}$ distance, which is in agreement with Haron et al., 1998. MBC, RMC in oil palm sole crop soil significantly decreased with increase in distance from palm base.

But in oil palm + cocoa soils, MBC, RMC was more in soils, which were farther away from palm base. However, the content of POXC varied with various distance from palm base, though, the variation was not significant. The soil AHC distribution was highest at $2 \mathrm{~m}$ distance from palm base. There was a significant difference between 1 and 4 $\mathrm{m}$ distance from palm base irrespective of seasons and soil depths. Our results agree with that of findings of Haron et al., 1998, who opined that dynamics of $\mathrm{C}$ fractions vary with time and space. The organic carbon in frond piles has been same in alleys and basins because of harvested leaves in alleys.
Studies on soil carbon dynamics indicated that the soil organic $\mathrm{C}$, water soluble $\mathrm{C}$, readily mineralizable $\mathrm{C}$, microbial $\mathrm{C}, \mathrm{KMnO}_{4}$ extractable $\mathrm{C}$, and acid hydrolysable carbon contents of two cropping systems varied significantly and highest values were recorded under oil palm + cocoa system. Higher contents of all the $\mathrm{C}$ fractions were observed in summer, while lower contents were seen during other seasons. Significant differences in soil carbon fractions were observed among three soil depths (0 to 15,15 to 30 and 30 to $60 \mathrm{~cm})$. All the carbon fractions decreased from surface to bottom soil layers. A linear increment in soil carbon fractions was observed with increase in distance from palm base.

\section{References}

Amanuel, W., Fantaw, Y. and Erik, K. 2018. Soil organic carbon variation in relation to land use changes: the case of Birrwatershed, upper Blue Nile River Basin, Ethiopia. Journal of Ecology and Environment, 42: 16.

Bhagya, H.P and Suresh, K. 2018. Carbon sequestration potential of oil palmcocoa cropping system grown in Andhra Pradesh under irrigated conditions. International Journal of Current Microbiology and Applied Science, 7(5): 358-362.

Blair, G.J. Lefroy, R.D.B. and Lise L. 1995. Soil carbon fractions based on their degree of oxidation, and the development of a carbon management index for agricultural systems. Australian Journal of Agricultural Research, 46: 1459-66.

Chan, K. W. 1976. A rapid method for studying the root distribution of oil palm and its application. In Proceeding International Development in Oil Palm, Earp, D. A. and Newall, W. ISP, Kuala Lumpur: 131-152. 
Cheshire, M.V. and Mundie, C.M. 1966. The hydrolyic extraction of carbohydrates from soil by sulfuric acid. Journal of Soil Science, 17: 372-381.

Don A., Schumacher J. and Freibauer A. 2011. Impact of tropical land-use change on soil organic carbon stocks-a meta-analysis. Global Change Biology, 17(4): 200-205.

Donia Jendoubi, Hanspeter Liniger, and Chinwe Ifejika Speranza. 2019. Impacts of land use and topography on soil organic carbon in a Mediterranean landscape (north-western Tunisia). Soil, 5: 239-251. doi.org/10.5194/soil5-239-2019.

Gomez, K. A, and Gomez, A.A. 1984. Statistical procedures for agricultural research, Second Edition, Wiley publishers pp. 704.

Haron, K., Brookes, P.C., Anderson, J.M. and Zakaria, Z.Z. 1998. Microbial Biomass and Soil Organic Matter Dynamics in Oil Palm (Elaeis Guineensis Jacq.) plantations, West Malaysia. Soil Biology and Biochemistry, 30(5): 547-552.

Kachaka, S., Vanlauwe, B. and Merckx, R. 1993. Decomposition and nitrogen mineralization of prunings of different quality. In: Mulongoy, K. and Merckx, R. (Eds.), Soil Organic Matter Dynamics and Sustainability of Tropical Agriculture. Wiley, Chichester pp. 199-208.

Lacerda-Júnior GV, Noronha MF, Cabral L, Delforno TP, de Sousa STP, Fernandes-Júnior PI, Melo IS and Oliveira VM . 2019. Land Use and Seasonal Effects on the Soil Microbiome of a Brazilian Dry Forest. Front. Microbiol. 10:648.doi: 10.3389/fmicb.2019.00648 122.

Lalith, M. R., Ranjith, P. U., Kristen, S. V., Shibu, J. and Salah, A. 2019. Cover Crop Influence on Soil Enzymes and
Selected Chemical Parameters for a Claypan Corn-Soybean Rotation. Agriculture, 9:125.

Mcgill, W.B., Cannon, K, R., Robertson, J. A. and Cook, F.D. 1986. Dynamics of soil microbial biomass and watersoluble organic $\mathrm{C}$ in Breton $\mathrm{L}$ after 50 years of cropping to two rotations. Canadian Journal of Soil Science, 66: 1-19.

Pan, C.M., Yang, F. and Zheng, H.S, 2000. Study on Soil Microbial Biomass in Rubber Plantation Intercropping with Amomum villosum or Coffea arabica. Soil and Environmental Science, 9: 114-116.

Shrinivas, N.S., Padmaja, P.S. and Krishnaraj, P.U. 2019. Chapter: Soil Metagenomics: Concepts and Applications. University of Agricultural Sciences, Dharwad, Karnataka, India. DOI: http://dx.doi.org/10.5772/intechopen.8 8958 .

Singh, I and Yadava, P.S. 2006. Spatial distribution of microbial biomass in relation to land-use in subtropical systems of north-east India. L. Tropical Ecology 47(1): 63-70.

Srivastava, S.C. and J.S. Singh.1991. Microbial biomass $\mathrm{C}, \mathrm{N}$ and $\mathrm{P}$ in dry tropical forest soils. Effect of alternate land uses and nutrient flux. Soil Biology and Biochemistry, 23: 117124.

Stefan Seiter, Elaine, R. I. and Ray, D. W. 1999. Dynamics of soil fungal and bacterial biomass in a temperate climate alley cropping system. Applied Soil Ecology, 12: 139-147.

Suresh, K. and Kiran Kumar, M. 2011. Carbon sequestration potential of oil palm under irrigated and rainfed conditions. Indian Journal of Dry land Agricultural Research and Development, 26(2): 55-57. 
Tailliez, B. 1971.The root system of Oil palm on the Sar Alberto Plantation in Columbia. Oleagineaux 26: 435-447.

Van der Mersch, M.K., Merckx, R. and Mulongoy, K. 1993. Evolution of plant biomass and nutrient content in relation to soil fertility changes in two alley cropping systems. In: Mulongoy, K. and Merckx, R. (Eds.), Soil Organic Matter Dynamics and Sustainability of Tropical Agriculture. Wiley, Chichester, pp. 143-154.

Vance, E.D., Brooker, P.C. and Jenkinso, D.S. 1987. An extraction method for measuring soil microbial biomass carbon. Soil Biology Biochemistry, 19: 703.

vanStraaten O, Corre MD, Wolf K,
Tchienkoua M, Cuellar E, Matthews RB, 2015. Conversion of lowland tropical forests to tree cash crop plantations loses up to one-half of stored soil organic carbon. Proc. Natl. Acad. Sci. USA, 112: 9956-9960.

Wakley, A. and Black, I.A. 1934. An examination of Degtjareff method for determining soil organic matter and a proposed modification of the chromic acid titration method. Soil Science, 37: 29-37.

Zhang, Y., Gu, F., Liu, S., Liu, Y. and Li, C. 2013. Variations of carbon stock with forest types in subalpine region of south western China. Forest Ecology and Management, 300: 88-95.

\section{How to cite this article:}

Manoja Katakam and K. Suresh. 2020. Soil Carbon Dynamics in Oil Palm - Cocoa Cropping System in Comparison to that of Oil Palm Sole Crop. Int.J.Curr.Microbiol.App.Sci. 9(03): 2452-2463. doi: https://doi.org/10.20546/ijcmas.2020.903.281 